\title{
MATERIA PRIMA Y PRIVACIÓN EN EL COMENTARIO TOMISTA A LA FÍSICA DE ARISTÓTELES'
}

\author{
Antonio Pérez-Estévez \\ Universidad del Zulia, Venezuela
}

\section{RESUMEN}

En los textos del Comentario a la Física de Aristóteles, Tomás de Aquino se esfuerza en destacar la distinción entre materia prima y privación. La materia prima es el sujeto del cambio sustancial; la privación es la carencia de forma que afecta a un sujeto de cambio, ya sea éste una sustancia natural ya sea la materia prima. Ambos son no-entes, es decir ambos están fuera del ámbito del ser formal o del ser en acto. Pero, mientras la materia prima es en potencia, la privación simplemente no-es. La materia prima posee una confusa entidad ubicada entre el ser en acto o ser formal y el no-ser; abarca al ámbito del ser en potencia. La privación simplemente no es y, en consecuencia, es solo un ente de razón, elaborado por la mente humana con fundamento en la realidad. La materia prima con su ser en potencia es el fundamento ontológico del incesante cambio en el universo y de la esencial temporalidad de toda sustancia individual.

Palabras claves: Tomás de Aquino, materia prima, privación.

\begin{abstract}
Thomas Aquinas in the text of his Commentary to Aristotle's Physics tries to point out the distinction between prime matter and privation. Prime matter is the subject of any substantial change; privation is the lack of a form affecting a subject of change, either a natural substance or prime matter. Both -prime matter and privation - are non-beings, that is, both are outside the scope of formal being or of being in act. But, if prime matter is in potency, privation simply is not. Prime matter owns a confused entity, located in between being in act or formal being and non-being; it belongs to the sphere of being in potency. Privation simply is not and, so, it is only a being of reason, built by human intellect with basement in reality. Prime matter with its being in potency is the ontological background of continuous change in the world and of the essential temporality of any individual substance.
\end{abstract}

Key words: Thomas Aquinas, Prime matter, Privation.

\section{INTRODUCCIÓN}

El ser natural se encuentra en continuo proceso de cambio o movimiento, es un continuo hacerse, fieri. El cambio es una de las experiencias primeras que tenemos en nuestro contacto con el mundo exterior. El árbol que ayer no tenía hojas, hoy las tiene; las hojas que ayer eran verdes, hoy son amarillas; la semilla de ayer, se ha convertido en árbol hoy; el niño de ayer,

1 Usamos como texto básico In Octo Libros Physicorum Aristotelis Expositio al que completamos con textos de In duodecim Libros Metaphysicorum Aristotelis Expositio y la Summa theologica. 
es hoy un joven apuesto y mañana un anciano encorvado que desaparecerá con la muerte en un próximo futuro para convertirse en ceniza o tierra.

Antes de Aristóteles, la explicación del hacerse en el cambio se había reducido o bien a negarlo y negar, a su vez, la multiplicidad de entes para afirmar que sólo existe un único ser inmóvil ${ }^{2}$ - Parménides - o bien a reducir la realidad a puro devenir o movimiento - Heráclito-. El devenir, decía Parménides, no puede provenir del ser, pues en ese caso ya sería antes de ser, lo que es contradictorio. Ni tampoco el devenir puede provenir de no-ser, pues del noser nada proviene. Heráclito, por otro lado, considera el devenir y el cambio como la única realidad existente, sin que parezca exigir en ese devenir un sujeto de cambio.

Aristóteles va a acercarse al hacerse en el cambio con una actitud analítica y científica. Dirá que en todo hacerse o cambio existe algo que cambia, el color en las hojas de un árbol, y algo sobre lo que se da el cambio y que permanece v.g. las hojas y el árbol, es decir, un sujeto o lo que está debajo del cambio. A lo que cambia, le llama forma - accidental o sustancial, según el caso- ya que en todo cambio desaparece una forma y nace una nueva; a aquello sobre lo que se da el cambio, lo llamará sujeto. Si la forma que cambia o se adquiere es accidental, el cambio será accidental, v.g. el color de las hojas de un árbol no modifica la sustancia del árbol. A este cambio accidental lo llama Tomás hacerse secundum quid, hacerse accidentalmente. Si la forma que se adquiere es una forma sustancial, el cambio será sustancial y se engendrará una nueva sustancia individual, como cuando una semilla se convierte en árbol. Tomás de Aquino llama al cambio sustancial, fieri simpliciter, hacerse simplemente ${ }^{3}$.En el cambio accidental, el sujeto de dicho cambio es una sustancia natural; en el cambio sustancial o en la generación de una nueva sustancia individual, el sujeto es la materia prima. Dos son, en consecuencia, los principios o causas per se de todo cambio: la forma y el sujeto ${ }^{4}$.

Pero el sujeto, aunque uno en número, es doble en su razón, debido a que en todo sujeto se da privación o carencia (de una forma futura que va a adquirir) lo que hace que el sujeto tienda a esa forma de la que carece hasta que la consigue, lo que supone un cambio o mutación ${ }^{5}$. En todo hacerse o en todo cambio aparece, en consecuencia, un tercer principio o causa, a saber, la privación o carencia. Aristóteles y Tomás de Aquino afirmarán que la privación no es un tercer principio per se del movimiento, sino sólo un principio per accidens, debido a que la privación es algo que le acaece al sujeto - accidit subjecto- como una suerte de accidente y que pierde en el momento del cambio cuando adquiere la nueva forma de la que está privado o carece ${ }^{6}$. Como consecuencia de lo dicho, tenemos que son dos los principios o causas per se del movimiento o cambio - el sujeto y la forma - y que hay un tercer principio o causa per accidens del cambio que es la privación o carencia de una forma que puede recibir.

El objetivo de nuestro trabajo es analizar el concepto que de materia prima, o sujeto de todo cambio sustancial, se desprende del texto del Comentario a los ocho libros de la Física

2 Tomás de Aquino, In Octo Libros Physicorum Aristotelis Expositio, Marietti, Turín-Roma, 1954, $\mathrm{n}^{\circ} 121$ : dixerunt enim quod nihil neque generatur neque corrumpitur: quod est contra veritatem et contra naturam....videbantur probari quod ens non generatur. Quia si ens fit, aut fit ex ente aut ex non ente: et utrumque horum videtur esse impossibile... quod enim ex ente aliquid fieri sit impossibile, et hoc manifestum est, quia id quod est non fit; nihil enim est antequam fiat: et ens iam est; ergo non fit. Quod etiam ex non ente aliquid fieri sit impossibile, ex hoc manifestum est, quia semper oportet aliquid subiici ei quod fit, ut supra ostensum est, et ex nihilo nihil fit... ut dicerent quod non essent multa entia, sed unum ens tantum.

3 In octo Libros Physicorum..., $\mathrm{n}^{\circ}$ 107: ...cum fieri dicatur multipliciter, fieri simpliciter est solum fieri substantiarum; sed alia dicuntur fieri secundum quid.

4 In Octo Libros Physicorum..., n 111: subjectum et forma sunt per se causae et principia omnis eius quod fit secundum naturam.

5 In octo Libros Physicorum..., $\mathrm{n}^{\circ}$ 105: Subjectum cui attribuitur fieri est duo ratione... in subjecto cui attribuitur fieri, est aliquid quod permanet et aliquid quod non permanet.

6 Op. cit., $\mathrm{n}^{\circ}$ 112: privatio vel contrarium est principium per accidens in quantum accidit subjecto. 
de Aristóteles y destacar, en contra de los platónicos, su diferencia entitativa de la privación. En consecuencia, el contenido del trabajo se dividirá en dos partes: 1) la materia prima: su incognoscibilidad y entidad; 2) privación y su diferencia de la materia prima; 3) conclusión.

\section{LA MATERIA PRIMA}

\subsection{La materia prima es incognoscible}

La gran dificultad que tuvieron Aristóteles y Tomás de Aquino para decir algo de la materia prima es precisamente su esencial incogniscibilidad. «La materia prima, por símisma, no puede ser conocida debido a que lo que conocemos lo conocemos por su forma» ${ }^{7}$. Tomás de Aquino, siguiendo a Aristóteles, sostiene que todo lo que conocemos, lo conocemos por su forma o, como dice en su Comentario a la Metafísica, el principio de todo conocimiento es la forma ${ }^{8}$. En consecuencia la materia prima, que carece de toda forma, no puede, en sí misma, ser conocida. Sin embargo, dirá que la conocemos por analogía o semejanza con lo que sucede cuando producimos artefactos como una silla o una cama. En todo hacerse o cambio sustancial, natural o artificial, aunque lo que conocemos sea la forma de la nueva sustancia individual, suponemos que tiene que haber algo, un sujeto, que recibe esta nueva forma sustancial. Así como la madera se convierte en una silla o en una cama cuando recibe la forma de silla o de cama, de manera semejante la materia prima se convierte en agua, en trigo o en perro, cuando recibe la forma de agua, de trigo o de perro. Tomás de Aquino añadirá que esta analogía por la que llegamos al conocimiento de la materia prima es una analogía de proporción, analogía secundum proportionem, es decir, que la relación proporcional existente entre una sustancia sensible como la madera y las formas artifíciales que pueda recibir como la forma de silla o de cama es semejante a la relación proporcional que existe entre la materia prima y las formas sensibles que puede recibir como son las formas de agua o de trigo o de perro?.

\subsection{Entidad de la materia prima}

La incognoscibilidad de la materia prima deriva de su escasa entidad metafísica. Tomás de Aquino, comentando al Estagirita, afirma que la materia prima no es uno en cuanto no es un individuo determinado, non sic unum est sicut hoc aliquid, hoc est sicut aliquod individuum demonstratum ${ }^{10}$. El individuo determinado o sustancia individual sensible es un compuesto ( $\sigma u \nu o \lambda o v)$ de materia y forma. La materia prima, sola, $(v \lambda \eta)$ no puede ser un individuo sustancial o una sustancia individual sensible, sencillamente porque la materia prima carece de toda forma. En el Comentario a los doce libros de la Metafisica de Aristóteles dirá que «sustancia» no puede predicarse unívocamente de la materia prima debido a que no posee las características propias de la materia prima, a saber: que sea separable o autónoma en su ser y que

7 Op. Cit., no 118: materia prima non potest sciri per seipsam, cum omne quod cognoscitur, cognoscitur per suam formam... sed scitur secundum analogiam, idest secundum proportionem.

8 In duodecim Libros Metaphysicorum Aristotelis Expositio, Turín-Roma, 1956, $\mathrm{n}^{\circ}$ 1296: (materia) secundum essentiam suam non habet unde cognoscatur, cum cognitionis principium sit forma.

9 Op. cit., $\mathrm{n}^{\circ}$ 1296: Cognoscitur autem per quamdam similitudinem proportionis. Nam sicut huiusmodi substantiae sensibiles se habent ad formam artificiales, ut lignum ad formam scamni, ita prima materia se habet ad formas sensibiles.

10 In octo Libros Physicorum..., n 118. 
sea un individuo determinado ${ }^{11}$. La materia prima ni es separable, es decir, no posee un ser autónomo ni es un individuo determinado sino justamente lo contrario: es inseparable de la forma sin la que no puede existir ${ }^{12}$, y es absolutamente indeterminada ya que la determinación la recibe de la forma en el compuesto sustancial. La sustancia puede decirse de la materia sólo 'concretive vel denominative', añade Tomás de Aquino, de manera semejante a como los accidentes se predican de la sustancia. Así como la sustancia es distinta por esencia de los accidentes así la materia prima por su esencia es distinta de las formas sustanciales que producen la sustancia individual ${ }^{13}$.

Si la materia no es una sustancia individual o un uno determinado, tampoco es uno de los nueve accidentes ya que el accidente es siempre una forma (accidental) que está inserta en una sustancia. Ni es sustancia ni es accidente o, como recoge de Aristóteles, «no es un algo o sustancia ni cualidad ni ninguno de los otros géneros en los que el ente se divide o se determi$n a{ }^{14}$. La materia prima no pertenece al ámbito o género de los entes. Pero posee alguna entidad. La materia prima explica «se dice ente y uno en cuanto está en potencia a la forma» ${ }^{15}$. Y esta disposición de la materia para estar en potencia a recibir el ser en acto o el ser formal, no es una propiedad o un accidente de la materia sino que constituye su esencia o sustancia. Esencia o sustancia por la que la materia prima está en potencia al ser o a la forma sustancial ${ }^{16}$. La materia prima, lo repetimos, es el sujeto sólo del cambio sustancial o de la generación de una nueva sustancia natural. La materia prima es un ente en razón de que está en potencia para recibir formas sustanciales. La materia prima es un ente en potencia, lo que significa que no es un ente propiamente dicho, sino que el ente se dice de la materia prima solo en razón de que está en potencia a la forma sustancial, que le da el ser en acto en la sustancia individual o compuesto. Ente, ser formal, ser en acto, son términos equivalentes en la ontología aristotélico-tomista ${ }^{17}$. Algo es un ente porque tiene ser formal es decir, porque es en acto y existe. La potencia de la materia prima, que constituye su esencia o sustancia, no es una potencia determinada a una forma sino que es una potencia o disposición indeterminada para recibir formas sustanciales, infinitas formas ${ }^{18}$. A la vez, y según la razón son muchas las potencias si tenemos en cuenta su relación a las diversas formas que puede recibir ${ }^{19}$.

Habría que recordar que ente, es aquello que posee el acto de ser, y se dice en primer lugar de la sustancia individual, debido a que ésta posee el acto de ser o existe. La materia no es, en

11 In duodecim Libros Metaphysicorum..., $\mathrm{n}^{\circ}$ 1291: impossibile est solam materiam esse substantiam...duo enim sunt, quae maxime propria videntur esse substantiae, quorum unum est, quod sit separabilia. Accidens enim non separatur a substantia, sed substantia postest separari ab accidente. Aliud est, quod substantia est hoc aliquid demonstratum. Alia enim genera non significant hoc aliquid.

12 Op.cit., $n^{\circ}$ 1292: materia enim non potest per se existere sine forma per quam est ens actu.

13 In duodecim Libros Metaphysicorum..., $\mathrm{n}^{\circ}$ 1289: non est enim intelligendum quod substantia actu existens (de qua hic loquimur) de materia praedicetur praedicatione univoca, sive quae est per essentiam...sed intelligendum est de denominativa praedicatione, per quem modum accidentia de substantia praedicantur... ipsa ergo concretiva sive denominativa praedicatio ostendit, quod sicut substantia est aliud per essentiam ab accidentibus, ita per essentiam aliud est materia a formis substantialibus.

14 In duodecim Libros Metaphysicorum..., n $\mathrm{n}^{\circ}$ 1285: dico autem materiam esse «quae secundum se idest secundum sui essentiam considerata nullatenus est «neque quid», idest neque substantia, «neque qualitas, neque aliquid aliorum generum, quibus ens dividitur vel determinatur».

15 In octo Libros Physicorum..., $\mathrm{n}^{\circ}$ 118: (materia) dicitur ens et unum inquantum est in potentia ad formam.

16 In octo Libros Physicorum...; n ${ }^{\circ} 131$ : Non igitur potentia materiae est aliqua proprietas addita super essentiam eius; sed materia secundum suam substantiam est potentia ad esse substantiale.

17 Pensamos que intencionalmente Tomás de Aquino, en este Comentario, evita hablar de la distinción de esencia (sustancia) - existencia con el fin de mantenerse en la concepción aristotélica de sustancia.

18 In duodecim Libros Metaphysicorum..., $\mathrm{n}^{\circ}$ 196: et haec est vera natura materiae (primae), ut scilicet non habeat actu aliquam formam, sed sit in potentia ad omnes.

19 In octo Libros Physicorum..., n $\mathrm{n}^{\circ}$ 131: Et tamen potentia materiae subjecto est una respectu multarum formarum; sed ratione sunt multae potentiae secundum habitudinem ad diversas formas. 
consecuencia, un ente propiamente dicho ya que no posee el acto de ser ni existe por sí misma. El ser en acto no lo tiene la materia prima sino que está en potencia o en capacidad de recibirlo de la forma en la sustancia individual o compuesto de materia y forma. El ser en potencia de la materia prima equivale a un no-ente secundum accidens. Las expresiones que usa Tomás de Aquino para describir la escuálida entidad de la materia prima -ente en potencia, no-ente per accidens - la tornan sumamente escurridiza. La materia es el único ente en potencia. Pero no es el único no-ente. De hecho en su Comentario a los 12 libros de la Metafisica $\left(\mathrm{n}^{\circ} 2437\right)$ señala textualmente:

Se dice no-ente de tres maneras. De una manera aquello que no es en modo alguno; y de tal no-ente no se produce la generación, porque de la nada nada se produce naturalmente. De otra manera se dice no-ente la privación, la cuál es pensada en un sujeto; y de tal no-ente ciertamente se produce la generación, pero por accidente, en cuanto que la generación se produce a partir de un sujeto al que le afecta una privación. De una tercera manera se dice no-ente la materia, la cuál, en sí misma, no es ente en acto sino ente en potencia. $Y$ de tal no-ente se produce la generación per $s e^{20}$.

Como vemos, existen tres maneras de predicar el no-ente, en una escala ascendente en razón de su mayor entidad, yendo de la nada absoluta a la materia prima pasando por la privación. Se dice no-ente, en primer lugar, la nada absoluta, de la que nada se puede producir. En segundo lugar, se dice no-ente la privación que viene a ser como una suerte de accidente en un sujeto (la materia prima), accidit subjecto, y de la privación se produce la generación pero sólo por accidente. Finalmente se dice no-ente de la materia prima en la medida en que, en sí misma, no es un ente en acto sino un ente en potencia. Y es a partir de la materia prima que la generación se produce per se. La materia, en consecuencia, es el no-ente con mayor entidad, aunque por su ser en potencia no alcance el status de ente o ser en acto. Y es sólo a partir de la materia prima que se produce per se la generación. A partir de la privación se produce la generación sólo por accidente.

De acuerdo a las precisiones que hemos hecho en torno a la mínima entidad de la materia prima, podemos deducir que el ser en potencia de la materia prima posee una menor entidad que todo ser formal o en acto, lo que significa que posee una entidad formal menor que el compuesto sustancial de materia y forma, menor también a cualquier forma sustancial e, incluso, menor que la entidad de cualquier accidente. El accidente, por su ser formal, posee un ser en acto, aunque accidental, y, en consecuencia, posee una mayor consistencia óntica que la materia prima. Esto explica que, para Tomás de Aquino, sea metafísicamente posible que un accidente exista sin su sujeto, como sucede milagrosamente en la Eucaristía, y sea absolutamente imposible que la materia prima exista sin forma alguna. En consecuencia, ni Dios en su omnipotencia, puede hacer que la materia prima exista sola sin la forma ${ }^{21}$.

20 In duodecim Libros Metaphysicorum..., $\mathrm{n}^{\circ}$ 2437: dicitur enim non ens tripliciter. Uno modo quod nullo modo est; et ex tali non ente no fit generatio, quia ex nihilo nihil fit secundum naturam. Alio modo dicitur non ens ipsa privatio, quae consideratur in aliquo subjecto; et ex tali non ente fit quidem generatio, sed per accidens, inquantum scilicet generatio fit ex subjecto, cui accidit privatio. Tertio modo dicitur non ens ipsa materia, quae, quantume est de se, non est ens actu, sed ens potentia. Et ex tali non ente fit generatio per se.

21 La materia prima es considerada como un no-ente, lo que significa que la materia prima no es un ente o un ser acto. Pero tampoco es un ente de razón, es decir, producido por obra de la mente humana, como sucede con lo que no es ente en la naturaleza v.g. las negaciones y las privaciones (Summa theologica, Ia-IIae, q. 8, a. 1, ad 3um). De hecho, la materia y la forma no pueden ser extrañas al ser, es decir, no pueden ser verdaderos no-entes o entes de razón (De Potentia, q. 7, a. 2, ad 9um). La entidad de la materia prima se ubica en una zona borrosa intermedia entre los entes propiamente dichos o seres en acto y los auténticos no-entes o entes de razón. (Ver a este respecto: Pérez-Estévez, A., «Entidad de la materia prima en Tomás de Aquino» en Veritas, vol. 48, No. 3). 


\section{LA PRIVACIÓN Y SU DISTINCIÓN DE LA MATERIA PRIMA}

Privatio, quae ponitur principium naturae per accidens, non est aliqua aptitudo ad formam, vel inchoatio formae vel aliquod principium imperfectum activum, ut quidam dicunt, sed ipsa carentia formae vel contrarium formae, quod subjecto accidit ${ }^{2}$.

Vemos que en esta definición que aparece en el Comentario a la Física de Aristóteles comienza por decirnos que es un principio de la naturaleza per accidens. En efecto, ya sabemos que los principios per se o esenciales de toda sustancia natural son dos: la materia y la forma. La privación es un principio natural per accidens, debido a que, a manera de accidente, afecta al sujeto a partir del cuál se origina la mutación. Digo a manera de accidente que afecta al sujeto, accidit subjecto, aunque no es ningún accidente. El accidente es una forma y es, en consecuencia, un ser en acto, aunque accidental. La privación, por el contrario, es carencia de forma y, por tanto, absoluto no-ser en acto $^{23}$. La privación es lo contrario de la forma, la noforma.

Pasa luego a decirnos que la privación no es una aptitud a la forma ni una incoación de la forma ni algún principio imperfecto activo. No es una aptitud o disposición o potencia pasiva a la forma ni ningún principio imperfecto activo como pudiera ser una incoación de la forma.

La materia prima es aptitud o disposición o potencia pasiva a la forma por la que puede recibir formas, infinitas formas. La privación no es ni siquiera eso: disposición o potencia pasiva a la forma.

Igual que la materia prima, la privación es carencia de forma. Pero, a diferencia de la materia prima, es lo contrario de la forma. La materia prima es un sujeto que carece de forma; la privación es lo contrario de la forma, la no-forma. La privación, igual que la forma, se encuentra en el sujeto, pero no como algo positivo e imperfecto sino sólo como carencia de forma. Es la pared, en cuando no verde, la que puede moverse y convertirse en verde, al recibir la forma de verde.

Ya hemos visto que la entidad de la privación se encuentra fuera del ámbito del ser formal, igual que la materia prima. Pero, si la materia prima es un no-ente per accidens, en el sentido de que es un ente en potencia, la privación es un no-ente per se, aunque no equivale a la nada absoluta o el absoluto no-ente. De la nada o del no-ente absoluto nada proviene y nada se engendra. De la privación, se engendra algo en el sentido de que algo se engendra a partir del sujeto en el que se da una privación o una carencia de forma. El sujeto del cambio puede ser: a) o bien una sustancia natural, en su carácter de privada de alguna forma, la que está en disposición de recibir una nueva forma. Es el cambio accidental; b) o bien la materia prima, por el hecho de carecer de toda forma, la que está en disposición de recibir una forma sustancial y convertirse en una sustancia natural individual. Es el cambio sustancial. La casa, en su carácter de no blanca, puede cambiar y convertirse en blanca, al recibir la forma accidental de la blancura. La madera, en su carácter de no ceniza, puede cambiar y convertirse en ceniza, al recibir la forma sustancial de ceniza.

Privatio est penitus non ens, cum privatio nihil aliud sit quam negatio formae in subjecto, et est extra totum ens ${ }^{24}$.

22 In octo Libros Physicorum Aristotelis Expositio, $\mathrm{n}^{\circ} 113$.

23 La idea de que la privación afecta al sujeto — sustancia natural o materia prima — a manera de accidente, es reiterativa. Así en In duodecim Libros Metaphysicam Aristotelis Expositio ( $\left.{ }^{\circ}{ }^{\circ} 1290\right)$ afirma que «las negaciones de las formas que son las mismas privaciones están en la materia secundum accidens», «negationes formarum quae sunt ipsae privationes, secundum accidens, insunt materiae».

24 In octo Libros Physicorum Aristotelis Expositio, $\mathrm{n}^{\circ} 135$. 
La privación se encuentra fuera del ámbito del ente, es absoluto no-ente. Comparada la entidad de la privación con la entidad de la materia, decíamos que la materia prima es un no-ente per accidens, en el sentido de que no es un ente formal o en acto pero puede ser o posee la disposición de recibir el ser en acto de la forma. Es un sujeto con disposición de recibir el ser en acto formal. En términos sencillos, decimos de la materia prima que su entidad real y metafísica es algo intermedio entre el ser en acto de la forma y el absoluto no ser o la nada. De la privación, decimos, que es absoluta carencia de ser que afecta a un sujeto y, en razón de esa carencia de ser formal, ese sujeto puede cambiar y convertirse en un sujeto distinto, ya sea accidentalmente, al recibir una nueva forma accidental, ya sea sustancialmente, al recibir una nueva forma sustancial.

Dentro del ámbito del no-ser formal, la privación se encuentra más próxima de la nada absoluta que de la materia prima. Ambas, la nada y la privación, son absolutos no-entes, aunque la nada es un absoluto y sustancial no-ente, mientras que la privación es un absoluto y accidental no-ente que afecta a un sujeto. De la nada, nada puede provenir o engendrarse. De la privación, en cuanto afecta a un sujeto material, puede provenir, accidentalmente, per accidens, un cambio accidental o una generación. Todo cambio y generación provienen de un sujeto, pero en cuanto afectado por una carencia o privación. Un sujeto, sin carencias o privaciones, no sufriría cambio o generación alguna.

«Ex non ente nihil fit simpliciter et per se, sed solum secundum accidens: quia quod est, idest ens, per se quidem non est ex privatione» 25 .

El ente o lo que es no proviene per se de la privación, ya que del no ente nada proviene simplemente o per se. De la privación, como ya hemos dicho, el ser proviene de manera accidental, en el sentido que el ente o lo que es proviene de un sujeto - materia prima o sustancia natural- afectada por una carencia o privación. El ser en acto o ente proviene per se de un sujeto afectado por una privación, es decir, carente de una forma que puede recibir. Cuando esa forma de la que carece, ese sujeto la adquiere, desaparece la privación, «privatio non intrat essentiam rei factae» ${ }^{26}$, para convertirse ese sujeto en un sujeto distinto, ya sea accidentalmente al recibir una forma accidental ya sea sustancialmente al recibir una forma nueva sustancial. La pared, en cuanto no blanca o carente de blancura, puede recibir la forma de blancura y convertirse en una pared blanca. La pared de no blanca ha pasado a ser blanca. La privación no forma parte esencial o per se de la naturaleza. De hecho, desaparece esta privación en el momento mismo en que un sujeto recibe la forma contraria. Pero, a la vez, una nueva privación afecta a este nuevo sujeto, en razón de la cuál, puede cambiar y recibir una nueva forma contraria a esa nueva privación. Todo sujeto natural, por su carencia o privación, está siempre en disposición de cambiar. Si ese sujeto natural es una sustancia, el cambio será un cambio accidental. Si, por el contrario, el sujeto natural es la materia prima, el cambio será sustancial, ya que la nueva forma recibida es una forma sustancial.

El sujeto, sustancia natural o materia prima, o aquello a partir de lo que se produce un cambio, permanece una vez que el cambio se ha producido o se ha recibido la nueva forma, ex hoc autem aliquid fit per se, quo inest rei postquam iam facta est. La sustancia natural o la materia prima, sujetos a partir de los que se produce el cambio, permanecen, una vez producido dicho cambio o recibida la nueva forma. La pared, sustancia natural, permanece una vez recibida la forma de blancura. La materia prima permanece una vez que la madera se ha convertido en ceniza. Son parte esencial del cambio y del nuevo sujeto v.g. la pared es parte esencial de la pared blanca y la materia prima es parte esencial de la ceniza. La materia prima, seme- 
jante a la madre que es causa de la generación en el recibir, es causa constituyente de toda cosa o sustancia natural en cuanto recibe la forma ${ }^{27}$.

Quod privatio pertineat ad malum, ostendii per hoc, quod forma est quoddam divinum et optimum et appetibile $e^{28}$.

La privación es el mal por ser lo contrario de la forma, es decir, la no-forma. La forma es algo divino, bueno y apetecible. Divino en cuanto es una participación de la semejanza del ser divino que es acto puro. Bueno y óptimo debido a que el acto es la perfección de la potencia y su bien. En consecuencia, es también apetecible, debido a que todo apetece su perfección y su bien ${ }^{29}$. La privación, en razón de ser lo contrario de la forma y la remoción de la forma, es decir, es lo contrario del bien y de lo apetecible e, incluso, remueve o elimina el bien. Es el mal. La materia, sin ser el bien como la forma, participa del bien en el sentido de que lo apetece, pues apetece sin descanso formas y tiende hacia ellas ${ }^{30}$.

\section{CONCLUSIÓN}

Siguiendo a Aristóteles, Tomás de Aquino, señala como principios o causas de todo cambio: el sujeto, la forma y la privación. El sujeto y la forma son causas per se del cambio. En todo cambio se da algo que cambia, es decir, la forma. $Y$ algo sobre lo que se da el cambio, a saber, el sujeto. Si la forma que cambia es accidental, el cambio será accidental y el sujeto de dicho cambio será una sustancia individual. Si la forma que cambia es sustancial, el cambio será sustancial y el sujeto de dicho cambio será la materia prima. La privación es la compañera inseparable de todo sujeto en el que se produce el cambio. Sin privación o carencia de la forma que va a recibir, no se produciría cambio alguno en el sujeto. Pero la privación es sólo causa o principio per accidens del cambio en el sentido de que el cambio de forma se produce en un sujeto, debido precisamente a que está privada o carece de la forma que va a recibir.

El sujeto es, por tanto, aquello a partir de lo que se produce el cambio en razón de la privación o carencia concreta de una forma. En el accidental, el sujeto a partir del que se produce el cambio es la sustancia individual en la que se da un cambio de formas accidentales. En el cambio sustancial o generación, el sujeto a partir del que se produce el cambio es la materia prima, la cual recibe una forma sustancial.

El texto del Comentario a los ocho libros de la Física se esfuerza en distinguir la materia prima de la privación para evitar la confusión en la que habían caído los platónicos. Tal distinción se advierte cuando se explicitan las escasas entidades de ambas.

La materia prima no es un esto o un individuo determinado, debido a que todo individuo es un compuesto de materia y forma. Sin forma, la materia prima no puede ser algo determinado ya que toda determinación proviene de la forma. Al no ser un individuo determinado y separable, la materia prima no puede ser una auténtica sustancia. Tampoco es ninguno de los

27 In octo Libros Physicorum..., $\mathrm{N}^{\circ}$ 135: ...materia, simul cum forma est causa eorum quae fiunt secundum naturam, ad modum matris: sicut enim mater est causa generationis in recipiendo, ita et materia.

28 In octo Libros Physicorum..., $\mathrm{N}^{\circ} 135$.

29 In octo Llibros Physicorum..., $\mathrm{N}^{\circ} 135$ : Quod privatio pertineat ad malum, ostendii per hoc, quod forma est quoddam divinum et optimum et appetibile. Divinum quidem est, quia omnis forma est quaedam participatio similitudinis divini esse, quod est actus purus: unumquodque enim in tantum est actu in quantum habet formam. Optimum autem est, quia actus est perfectio potentiae et bonum eius: et per consequens sequitur quod sit appetibile, quia unumquodque appetit suam perfectionem. Privatio autem opponitur formae, cum non sit aliud quam remotio eius: unde cum id quod opponitur bono et removet ipsum, sit malum. Manifestum est quod privatio pertinet ad malum

30 Summa theologica, $\mathrm{I}^{\mathrm{a}}, \mathrm{q}$. 5, a. 2, ad lum: Materia prima participat bonum, cum appetat ipsum (nihil autem appetit nisi simile sibi): non autem participat ens cum ponatur non-ens. 
nueve accidentes posibles. En consecuencia, la materia prima no es propiamente un ente. La entidad de la materia prima consiste en su ser en potencia o en su disposición a recibir formas, infinitas formas. Ahora bien, el ente es ente en razón de su ser-en-acto. Por eso, la materia prima, que es sólo ser-en-potencia, no es propiamente ente. De hecho, Tomás de Aquino lo considera en distintos textos como uno de los no-entes. Un no-ente con mayor entidad que la privación y que el absoluto no-ser o la nada.

La materia prima no es un ente o ser en acto, es decir, sola, sin forma, ni existe ni puede existir, se encuentra fuera del ámbito del ser formal. La materia prima es un no-ente formal pero es en potencia. La potencia de recibir infinitas formas no es algo accidental que la materia prima pueda o no poseer. La potencia de recibir formas es la esencia misma de la materia prima lo que significa que la entidad de la materia se reduce a su ser en potencia o, como dice textualmente, se dice ente y uno en cuanto está en potencia a la forma. Su ser en potencia para recibir formas, le permite ser el sujeto del cambio sustancial. Es a partir de la materia prima que se origina todo cambio sustancial o la generación de una nueva sustancia individual.

También la privación es un no-ente, porque no es un ente en acto o un ente formal. Tampoco la privación existe ni puede existir. Pero la privación ni siquiera es en potencia. La privación es la no-forma, es decir, es la carencia de una forma que un sujeto va a recibir. La materia prima es un sujeto sin forma; la privación es la no-forma, lo contrario de la forma. Si la materia prima no es un ente en acto o formal pero es en potencia, la privación simplemente no es o es un absoluto no ente. La materia prima posee algún tipo de entidad, oscura y escurridi$\mathrm{za}$, que sin llegar a ser en acto o a existir es en potencia. La entidad de la materia prima se ubica entre el ser en acto y el absoluto no-ser; su entidad es ser en potencia, lo que le permite ser el sujeto de todo cambio sustancial o generación. La privación no posee entidad alguna real; es sólo un ente de razón o la elaboración mental de una carencia de ser atribuida a un sujeto ${ }^{31}$. La materia se encuentra fuera del ámbito del ser formal o de la existencia pero pertenece al ámbito del ser en potencia. La privación está fuera de todo ámbito de ser. Simplemente no es.

La materia prima, en cuanto sujeto del cambio sustancial o generación, se introduce en la nueva sustancia que se engendra. Constituye, con la forma sustancial, el ser del nuevo individuo sustancial. La privación, por el contrario, no forma parte de la nueva entidad que se produce en todo cambio. La privación o carencia de la forma a recibir, desaparece justamente cuando se da el cambio o cuando el sujeto recibe la nueva forma. La pared no-blanca que se convierte en blanca, pierde su privación de blancura en el preciso instante en que se convierte en blanca. La materia prima que se convierte en un árbol, pierde su privación de no-árbol en el preciso momento en que se convierte en árbol. Es cierto que el nuevo ser que resulta del cambio adquiere nuevas y concretas privaciones que lo disponen a futuros cambios, pero pierde la privación o carencia de forma que tenía antes del cambio recién producido. La privación, en fin, es la carencia de una forma que afecta a un sujeto, accidit subjecto, mientras que la materia prima es el sujeto de todo cambio sustancial. La privación es compañera inseparable de la materia prima, pero ciertamente no es la materia prima.

Es preciso también distinguir la privación de la potencia de la materia prima con la que tiene mucha analogía. La potencia infinita e indeterminada de la materia prima entraña disposición indeterminada de la materia prima para recibir infinitas formas. La privación es carencia determinada de una forma concreta que afecta a un sujeto - materia prima o sustancia individual-. La potencia, como esencia de la materia prima, no se aleja jamás de ella. La privación, como carencia determinada de una forma concreta, desaparece en el preciso instante en que dicha forma es recibida por el sujeto. La potencia de la materia prima parece ser el fundamento ontológico de todas las privaciones concretas que irán afectando a una sustancia individual y que la hacen esencial e incesantemente cambiante. Sin el ser en potencia de la ma- 
teria prima, no podrían darse las privaciones determinadas ni explicarse el cambio incesante del universo. El ser en potencia de la materia prima es el fundamento ontológico de que toda sustancia individual se sienta afectada de una radical carencia que la conduce a un cambio incesante y a una esencial temporalidad.

Antonio Pérez-Estévez Apartado 10.114 Maracaibo Venezuela 